\title{
Determining the Effect of Neglecting Capillary Pressure on Fractional Flow by Numerical Analysis
}

\author{
Edwin Tan Jun $\mathrm{Yi}^{*}$ and Hamed Hematpur \\ Faculty of Engineering, Universiti Teknologi Petronas, Tronoh, Perak, Malaysia
}

\begin{abstract}
The fractional flow equation is simplified by neglecting the effect of capillary pressure gradient. However, zero capillary pressure assumption may induce error in the fractional flow equation. The effect of different parameters on capillary pressure gradient in fractional flow is determined with numerical analysis based on the saturation distribution profile. The fractional flow equation is dependent on the relative permeability and relative permeability is a function of saturation. This project presents one- dimensional black oil simulation in core flooding using gas-water system to compare the saturation profile with capillary pressure and without capillary pressure. A factorial design was established for four (4) different parameters, i.e., porosity, permeability, length and injection rate, in three (3) levels (34=81). Therefore, eighty-one (81) simulations were conducted and the results were analyzed via Design of Experiments. This study found that porosity, permeability and injection rate has visible effect in the saturation profile due to the negligence of capillary pressure. Due to the limitation of the simulator, the end capillary effect was not captured in this study. Hence, the capillary pressure has no visible effect towards the core length.
\end{abstract}

Keywords: Fractional flow; Capillary pressure gradient; Relative permeability; Two-phase flow

\section{Introduction}

Reservoirs are rarely homogenous. The effect of neglecting the capillary pressure in fractional flow approach faced more problems in heterogeneous properties, multiple spatial dimension and temporal changes. Although advection flow is highly dominated, diffusion flow existed in some parts of the reservoir. Capillary pressure is a function of pore size distribution factor which is affected by porosity and permeability. In addition, the capillary pressure gradient in the fractional flow equation is influenced by several parameters such as porosity, permeability, length and injection rate. The objective of this project is to study the effect of the above-mentioned parameters on the induced errors by zero capillary pressure assumption in fractional flow.

The scope of this study involved the fundamentals of reservoir engineering such as multiphase flow, immiscible displacement and horizontal displacement. In addition, the project focused on mathematical simulation which is by using one-dimensional black oil simulation. The result is dependent on the usage of literature correlations and equations. Most of the theoretical and empirical correlations are in accordance to limitations and assumptions. The utilization of software such as MATLAB computation software, ECLIPSE reservoir simulation software and Minitab statistical software were used to simulate and generate the results. For instance, the relative permeability profiles and capillary pressure profiles are generated based on Brooks- Corey correlation using MATLAB. ECLIPSE is used to generate the saturation profile through one-dimensional black oil simulation in core flooding by gas-water drainage process. The Design of Experiment (DOE) was used to establish the factorial design based on different parameters in multiple levels and the responses were analyzed.

\section{Conceptual Framework}

\section{Fractional flow concept}

A well-established theory developed by Buckley-Leverett known as the Frontal Displacement theory is able to determine the displacement of immiscible fluid in porous medium [1]. Morel-Seytoux states that the flow of air and water in unsaturated soils can be viewed as a multi-phase system and proposed that this concept can aid the Petroleum Engineers [2]. However, the viscosity of the fluid should be considered. Therefore, the concept proposed by Morel-Seytoux might not be applicable for oil- water system due to the high difference in viscosity ratio. Buckley-Leverett presented two equations which are the Fractional Flow Equation (1) and Frontal Advance Equation (6).

$$
f_{g}=\frac{1+\frac{k k_{r w} A}{q_{r} \mu_{w}}\left(\frac{\partial P_{c}}{\partial x}-\Delta \rho g \sin A\right)}{1+\frac{\mu_{g} k_{r w}}{\mu_{w} k_{r w}}}
$$

Fractional Flow Equation is derived based on Darcy Equation. The Darcy Equation is the standard mathematics tools for the petroleum engineers to determine the fluid flow. Assuming that an incompressible fluid is flowing linearly in a horizontal core sample with cross sectional area $(A)$ and length $(L)$, then the fluid flow equation is expressed as Darcy Equation (2) [3].

$$
q=\frac{k A \Delta P}{\mu \Delta x}
$$

The fractional flow is a ratio of fluid flow in multiphase phase condition. Ahmed (2010) suggested that Equations (3) and (4) below indicates that when gas is displacing the water, the increment in $f g$ at any point will result in corresponding decrease in $f w$ [3].

$$
F_{g}+f_{w}=1
$$

"Corresponding author: Edwin Tan Jun Yi, Faculty of Engineering, Universiti Teknologi Petronas, Tronoh, Perak, Malaysia, Tel: +60 5-368 8000; Fax: +60 5-365 4088; E-mail: edwinjunyi@gmail.com

Received May 29, 2017; Accepted June 30, 2017; Published July 06, 2017

Citation: Yi ETJ, Hematpur H (2017) Determining the Effect of Neglecting Capillary Pressure on Fractional Flow by Numerical Analysis. J Pet Environ Biotechnol 7 334. doi: 10.4172/2157-7463.1000334

Copyright: (C) 2017 Yi ETJ, et al. This is an open-access article distributed under the terms of the Creative Commons Attribution License, which permits unrestricted use, distribution, and reproduction in any medium, provided the original author and source are credited. 


$$
F_{g=} 1-f_{w}
$$

And by neglecting the capillary pressure gradient and assume the fluid flow in a horizontal direction, the fractional flow equation is simplified as shown in Equation (5).

$$
f_{g}=\frac{1}{1+\frac{\mu_{g} k_{r w}}{\mu_{w} k_{r g}}}
$$

The frontal advance Equation (6) could be used to obtain the saturation distribution across the distance analytically. Based on Equation (6), fractional flow equation is a function of saturation.0

$$
x=\frac{5.615 W_{i n j}}{\phi A} \frac{d f_{w}}{d S_{w}}
$$

In Figure 1, the saturation profile showed two distinct saturations at each distance. This phenomenon is physically impossible and occurs due to the negligence of capillary pressure.

Figure 2 shows that a displacement front was proposed to rectify the discontinuous saturation profile by balancing the area ahead of the front and below the curve. This solution provides an ideal piston-like displacement [1].

\section{Relative permeability and capillary pressure profile}

Fractional flow is a function of mobility ratio which requires the relative permeability profile. In this project, gas is the non-wetting phase, whereas, water is the wetting phase. The relative permeability for gas-water system is expressed in Equation (7) and (8) [4]. Figure

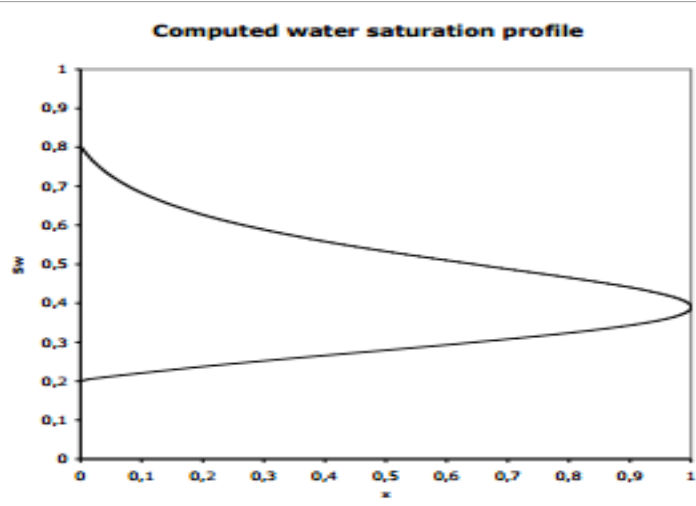

Figure 1: Computed water saturation profile [1].

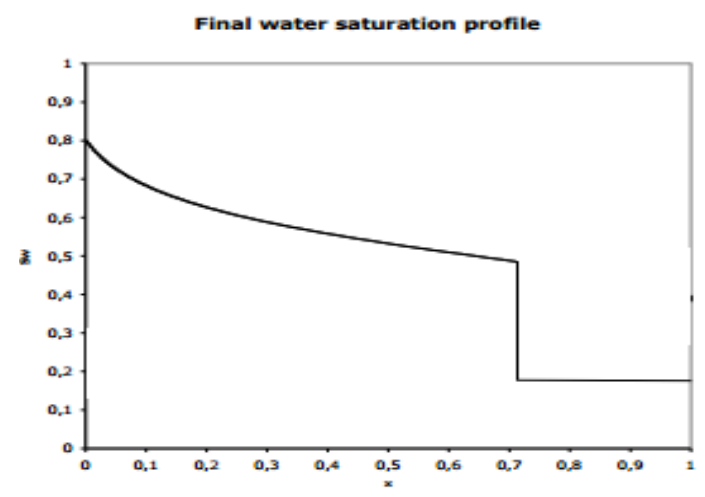

Figure 2: Final water saturation profile [1].

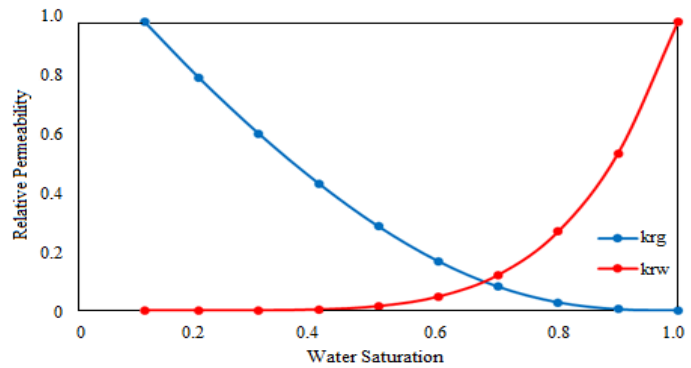

Figure 3: Relative permeability profile.

3 shows an example of relative permeability profile generated using equation (7) and (8). It is a water-wet system which is in line with typical sandstone rock properties.

$$
\begin{aligned}
& k_{r w}=\left(\frac{S_{w}-S_{w r}}{1-S_{w}}\right)^{\frac{2+3 \lambda}{\lambda}} \\
& k_{r g}=\left(\frac{1-S_{w}}{1-S_{w}}\right)^{2}\left(\frac{S_{w}-S_{w r}}{1-S_{w}}\right)^{\frac{2+3 \lambda}{\lambda}}
\end{aligned}
$$

The residual water saturation is a function of porosity and permeability as shown in equation (9) [5]. However, this correlation is limited to low permeability range only.

$$
S_{w r}=3.5 \frac{\phi^{1.26}}{k^{0.35}}
$$

The arrangement of the pore size in the porous medium is important in characterizing the properties of the medium. The rock pores can be determined in terms of pore size distribution factor. Huet combined previous relative permeability models and proposed a correlation. Equation (10) shows the correlation for pore size distribution factor ( $\lambda$ ) [6].

$$
\lambda=0.0098 \frac{k^{0.3792} P_{c \text { entry }}{ }^{0.6698}}{\phi^{0.6341}\left(1-S_{w r}\right)^{0.6835}}
$$

Equation (11) suggests the correlation for calculating the threshold capillary pressure or known as capillary entry pressure ( Pc entry).

$$
P_{c \text { entry }}=\text { Conversion factor } \times \frac{640.0538 \phi^{0.821}\left(1-S_{w r}\right)^{0.8486}}{k^{0.5285}}
$$

The function of is based on experiment using mercury and air as a two-phase system. Therefore, a conversion factor is needed to pressure of mercury-air could be converted to water-air capillary pressure using Equation (12).

$$
\frac{P_{c \text { water-air }}}{\sigma_{\text {water-air }} \cos \theta_{\text {water-air }}}=\frac{P_{c H g-a i r}}{\sigma_{H g-a i r} \cos \theta_{H g-a i r}}
$$

The capillary pressure profile is generated using equation (13) [4].

Figure 4 shows an example of capillary pressure profile generated using equation (13). The profile suggests that the capillary pressure increases when the water saturation is close the residual water saturation $(S w r)$.

$$
P_{c}=P_{c \text { entry }}\left(S_{w}^{*}\right)^{\frac{1+\lambda}{\lambda}}
$$


Capillary pressure is depending on the saturation. By implementing chain rule, the capillary pressure gradient is defined in terms of saturation gradient as shown in Equation (14).

$$
\frac{\partial P_{c}}{\partial x}=\frac{\partial P_{c}}{\partial S_{g}} \frac{\partial S_{g}}{\partial x}
$$

\section{Fluid flow in porous media}

Mass cannot be created or destroyed. Based on conservation of mass, the Diffusivity Equation (15) is derived based on Continuity Equation and Transport Equation.

$$
\frac{\partial^{2} P}{\partial x^{2}}=\phi C_{t} \frac{\mu}{k} \frac{\partial P}{\partial t}
$$

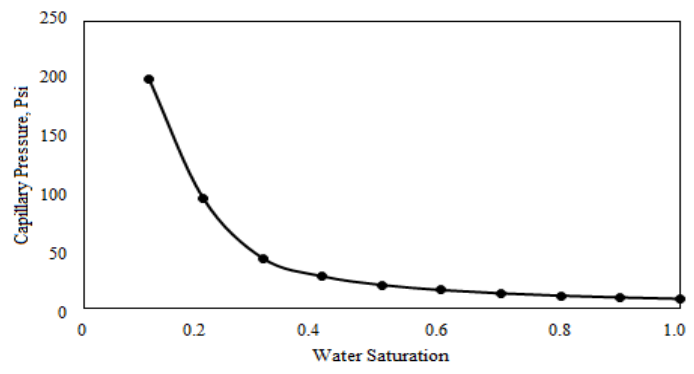

Figure 4: Capillary pressure profile.

\begin{tabular}{|c|c|}
\hline Materials & Porosity \\
\hline Sandstone & $0.02-0.40$ \\
\hline Clay & $0.01-0.18$ \\
\hline Limestone & $0.00-0.36$ \\
\hline
\end{tabular}

Table 1: Range of effective porosity [7].

\begin{tabular}{|c|c|}
\hline Rock Type & Permeability (mD) \\
\hline Oil Reservoir Rock & $100-10000$ \\
\hline Fresh Sandstone & $1-10$ \\
\hline
\end{tabular}

Table 2: Permeability chart by Bear in 1972.

\begin{tabular}{|c|c|c|c|}
\hline Parameters & Low & Middle & High \\
\hline Porosity & 0.1 & 0.25 & 0.4 \\
\hline Permeability (mD) & 5 & 250 & 500 \\
\hline Length (ft) & 0.3 & 1 & 1.7 \\
\hline Flow Rate (ft3 3 Day) & 0.0122 & 0.0305 & 0.0488 \\
\hline
\end{tabular}

Table 3: DOE (Design of Experiment) framework.

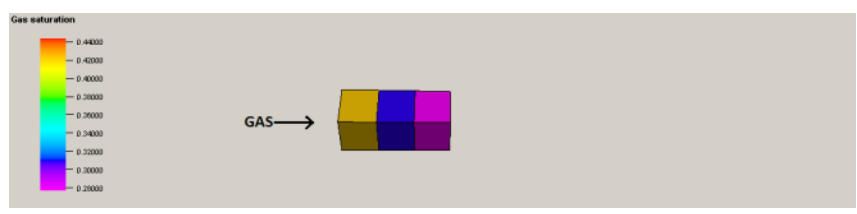

Figure 5: Three grid cells representing 0.3 feet core.

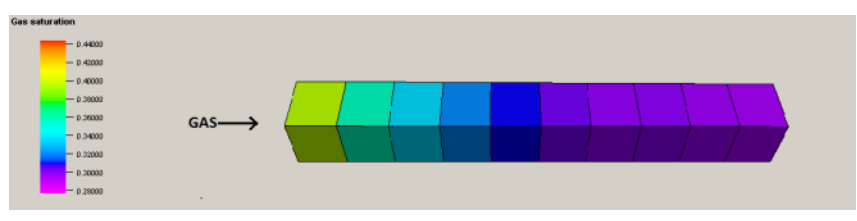

Figure 6: Ten grid cells representing 1.0 feet core.
The saturation distribution across the grid cells were calculated with the diffusivity equation.

\section{Research Methodology}

\section{Data gathering and analysis}

Table 1 suggested the range of effective porosity for sandstone is between $2 \%$ to $40 \%$ [7] which will be used in the simulations.

In Table 2, the range of permeability for sandstone and typical reservoir rock range from $1 \mathrm{mD}$ to $10000 \mathrm{mD}$ [8]. To account for limitation in Equation (9), the range of permeability was reduced to 25 $\mathrm{mD}$ to $500 \mathrm{mD}$.

The range for the core length was referred based on the core service provider in the petroleum industry which is between 3 inches to 24 inches. The flow rate was decided in line with the range of fluid velocity in the reservoir which commonly is 1 to 4 feet per day.

\section{Design of Experiment (DOE)}

A factorial design was established based on the framework decided as shown in Table 3. Sensitivity analysis was performed on four parameters which are porosity, permeability, length and flow rate in three levels (34=81).

Therefore, there are eighty-one sensitivity cases used to study the effect of the above- mentioned parameters on capillary pressure.

\section{Define simulation cases}

To define the simulation, the initial fluid PVT properties and rock physics function were kept constant. The relative permeability and capillary pressure profile were input based on each case parameter. The rock was assumed to be homogenous and all the grid cells have the same dimension. The grid cells were only connected in $\mathrm{x}$-direction to simulate the core flooding process.

Figures 5-7 shows three different models set up for different core lengths which are 1.7 feet, 1.0 feet and 0.3 feet, respectively. Gas was injected as indicated by the arrow shown. The time step chosen for all the cases are fixed at four pore volume injection. At four pore volume injection, the saturation distribution across the grid cells were charted and tabulated.

\section{Measure the responses}

After completion of simulation, the simulator output a saturation distribution profile across the grid cells for each case. Each case should have a trend line with capillary pressure and a trend line without capillary pressure.

Figure 8 shows a case example of saturation distribution with considering capillary pressure and without considering capillary pressure. This profile was generated for all eighty-one sensitivity cases.

Fractional flow profile is a function of saturation profile. Therefore, the saturation profile is able to represent the effect in the fractional flow profile.

The percentage error is calculated by comparing the saturation profile with capillary pressure and without capillary pressure. This study performs sensitivity analysis on four parameters which are length, permeability, porosity and flow rate. By measuring the difference in the saturation profile, the percentage error is able to be identified and analyzed using Design of Experiments (DOE). 


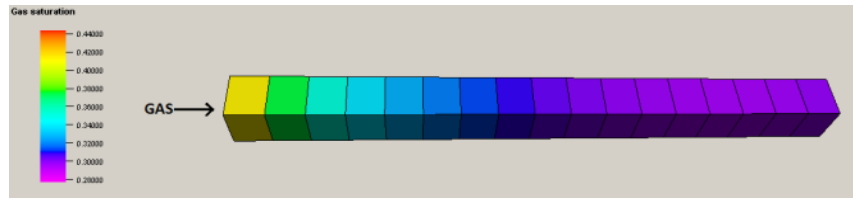

Figure 7: Seventeen grid cells representing 1.7 feet core.

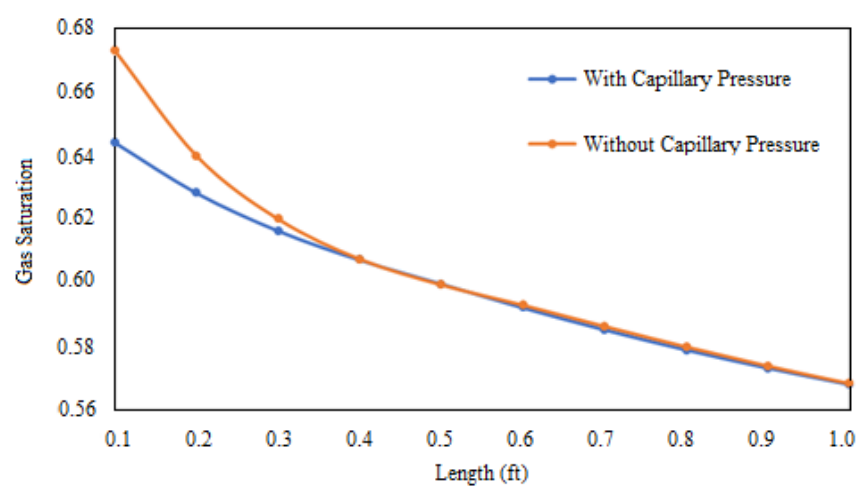

Figure 8: Saturation profile.

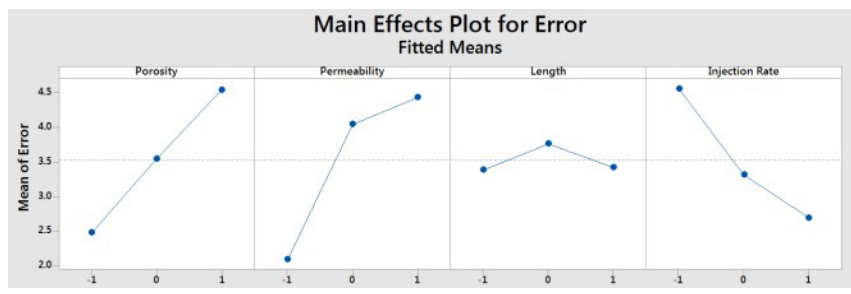

Figure 9: Error induced by porosity, permeability, length and injection rate.

$$
\text { Error }=\frac{\sum a b s\left\lfloor S_{g\left(P_{c}\right)}-S_{g\left(n o P_{c}\right)}\right\rfloor}{\sum S_{g\left(n o P_{c}\right)}} \times 100
$$

\section{Results and Discussion}

Figure 9 shows the result generated by DOE, the porosity and permeability is proportional with the induced error. However, injection rate is inversely proportional with the induced error, whereas, the length of the core does not show significant effect in terms of capillary pressure.

\section{Error induced by porosity}

Based on Geiser, the fluid velocity is related to the Darcy flux by the porosity as suggested in Equation (17) [9]. The flux is divided by porosity to account for the fact that only a fraction of the total formation volume is available for flow. Assuming at constant injection rate, the Darcy velocity varies according to porosity. Porosity represents the pore volume of the core. Lower the porosity, the higher the fluid velocity. Whereas, higher porosity will result in lower velocity and the effect of capillary pressure will be significant. It explains the increment in percentage difference as the porosity increases.

$$
\text { Actual velocity }=\frac{\text { Darcy Velocity }}{\phi}
$$

\section{Error induced by permeability}

The higher the permeability, the easier for the fluid to flow.
Therefore, the higher the permeability, the lower the capillary pressure gradient. On the contrary, based on the mathematical point of view, the fractional flow equation suggests that the permeability has inversely proportional relationship with the capillary pressure gradient. The fractional flow equation is a highly non-linear equation because the capillary pressure gradient is function of permeability and porosity.

According to Equation (18), the numerator suggests that the capillary pressure gradient and permeability will have inverse magnitude and the multiplication supposed to neutralize each other. Although the capillary pressure gradient increases exponentially but in order for the gas saturation to reach the residual water saturation, the pressure required is close to infinity. In this core flooding simulation, the average gas saturation that was injected into the core is lower than $70 \%$. Therefore, the capillary pressure gradient is relatively small and permeability will be dominant in the equation. As a result, the increment in permeability will create larger percentage difference due to the parameters' relationship in the equation.

$$
f_{g}=\frac{1+\frac{k k_{r w} A}{\mu_{w} q_{t}}\left(\frac{\partial P_{c}}{\partial x}\right)}{1+\frac{\mu_{g} k_{r w}}{k_{r g} \mu_{w}}}
$$

\section{Error induced by length of core}

In this case study, the result shows that length is not affected by capillary pressure gradient. The effect of induced error by the core length is not visible. In this project, the time step taken is at four pore volume. Gas already breakthrough and the gas saturation is distributed across the grid cells. The effect of saturation gradient will be high if the gas saturation has not reach the end of the core. In the simulation, the saturation gradient is similar across the grid cells due to the presence of gas saturation in all grid cells.

However, in actual experiment, the presence of capillary end effect needs to be accounted for. Capillary end effects emerge due to the discontinuity of capillarity in the wetting phase at the outlet of the core. The effect is prominent in drainage process where the wetting phase is being displaced by non-wetting phase such as gas displacing water [10]. Based on experimental outcome, longer core should be used to minimize the end effect. In the simulation, the end capillary effects are not visible.

\section{Error induced by injection rate}

Higher injection rate will result in higher fluid velocity and intensify the viscous force. Viscous force could be defined as the product of fluid velocity and the fluid viscosity $(\mu)$ as suggest by Equation (19). Capillary number is a ratio of viscous force over capillary force as stated in Equation (20). Capillary numbers are usually large for high speed flows. Therefore, the viscous force will be more dominant and is able to overcome the capillary force.

In addition, in high gas injection rate, the gas saturation across the grid is almost uniform. As suggested by the DOE result, for high injection rate, the percentage difference in the saturation profile for with capillary pressure and without capillary pressure is low.

\section{Conclusion}

Based on the design of experiments, the outcome for this study shows that the main error effects were induced by the permeability, porosity and flow rate. The core length is not significant in affecting the saturation profile. 
Citation: Yi ETJ, Hematpur H (2017) Determining the Effect of Neglecting Capillary Pressure on Fractional Flow by Numerical Analysis. J Pet Environ Biotechnol 7: 334. doi: 10.4172/2157-7463.1000334

Page 5 of 5

Error of fractional flow $=f(k, \varphi, q)$.

\section{Acknowledgements}

I would like to thank Department of Petroleum Engineering, Universit Teknologi Petronas for providing technical and logistics support. Besides that, my heartfelt gratitude to my supervisor and Associate Professor, Dr. Syed Mohammad Mahmood for his dedicated guidance. A special thanks to my mentor, Mr Hamed Hematpour for his encouragement. Furthermore, I would like to express my appreciation to my friends and family that provide moral support.

\section{References}

1. Buckley S, Leverett M (1942) Mechanism of fluid displacement in sands Transactions of the AIME 146: 107-116.

2. Morel-Seytoux H (1973) Two-phase flows in porous media. Advances in Hydroscience 9: 119-202.

3. Ahmed TH (2010) Reservoir engineering handbook. (4th edn) Gulf Professional Publications, Elsevier/GPP, Amsterdam.

4. Brooks RH, Corey AT (1964) Hydraulic properties of porous media and their relation to drainage design. Transactions of the ASAE 7: 26-28.

5. Timur A (1968) An investigation of permeability, porosity and residual water saturation relationships for sandstone reservoirs. Society of Petro-physicists and Well-Log Analysts, The Log Analyst. 9:4.

6. Huet CC, Rushing JA, Newsham KE, Blasingame TA (2005) A modified Purcell/ Burdine model for estimating absolute permeability from mercury-injection capillary pressure. Data International Petroleum Technology Conference, Doha, Qatar.

7. McWhorter DB, Sunada DK (2010) Ground-water hydrology and hydraulics. Water Resources Publications, LLC, Colorado, USA.

8. Dietrich P, Helmig R, Sauter M, Hötzl H, Köngeter J (2005) Flow and transport in fractured porous media. Springer, Berlin.

9. Geiser J, Noack L (2010) Iterative operator-splitting methods for nonlinear differential equations and applications of deposition processes. E-doc Publication, Humboldt-University, Berlin. 27: 1026-1054.

10. Huang DD, Honarpour MM (1998) Capillary end effects in core flood calculations. J Pet Sci Eng 19: 103-117. 